\title{
Intelligent Allocation of Network Bandwidth: A Comparison of Two Generalized Particle Models
}

\author{
Xiang Feng \\ Department of Computer Science \\ East China University of \\ Science and Technology \\ Shanghai, China 200237 \\ Email: xfeng\{@ecust.edu.cn, @cs.hku.hk\}
}

\author{
Francis C.M. Lau \\ Department of Computer Science \\ The University of \\ Hong Kong, Hong Kong \\ Email: fcmlau@cs.hku.hk
}

\author{
Dianxun Shuai \\ Department of Computer Science \\ East China University of \\ Science and Technology \\ Shanghai, China 200237 \\ Email: shdx411022@online.sh.cn
}

\begin{abstract}
There are two types of generalized particle models (GPM) that can be used to allocate network bandwidth efficiently. One is the "original" generalized particle models (OGPM) [1] with which the allocation is not changed until the price reaches equilibrium. The other one is the economic generalized particle model (EGPM) [2], where the allocation is changed dynamically even when the allocation is being calculated. EGPM therefore suits bandwidth allocation in dynamic environments. The two models can be classified as evolutionary intelligent swarm optimization approaches. The EGPM is an important extension and further development of the OGPM, which comprises two major components: (1) dynamic allocation of network bandwidth based on GPM; and (2) dynamic modulation of price and demands of network bandwidth. The two components combined can be readily implemented by a distributed algorithm. In this paper, by simulations, we compare EGPM and OGPM, and then with the ant colony optimization approach, the utility function algorithm, and the max-min fairness algorithm.
\end{abstract}

Index Terms - Intelligent bandwidth allocation, evolutionary swarm optimization, generalized particle models (GPM), economic generalized particle model (EGPM), distributed and parallel algorithm, dynamical process.

\section{RELATED WORK}

In [1], Shuai and Feng proposed the generalized particle model for solving optimization problems, which we refer to as the original generalized particle model (OGPM) in this paper. OGPM is based on hybrid energy functions. It has overcome some of the main deficiencies while retaining the good features of the Lagrangrian multiplier approach, the maxmin algorithm, and ant colony algorithm. Briefly, OGPM

- is easy to realize;

- can achieve comparatively higher network resource availability; and

- can operate with a decentralized control.

During the last several years, different approaches using economic models for resource allocation have been proposed [13-19]. Some of them divide the traffic into multiple priority classes, but use fixed prices for each service class. By adding congestion-dependent components into the price yields a dynamic pricing strategy. Such a strategy would take network activities into account, and can improve network efficiency by offering more competitive prices.
Wang [3] proposed a strategy where the price depends on the service class's average demand. Specifically, the price is negotiable through a negotiation protocol. However, the strategy requires resource reservation in the network, which can raise a few key issues such as inefficient use of the network, increased network cost, and most importantly impractical use in real time.

Baglietto et al. gave a pricing model for Best Effort (BE) service by assigning the same amount of bandwidth to all classes of traffic [4]. Equivalent bandwidth allocation for each type of traffic class is not a reasonable strategy though it makes the approach simple. Pricing based on equivalent bandwidth is not fair for customers needing different treatments.

The scheme proposed in [5] used price to reflect the resource demand and supply situation. Pricing is worked out under a well-defined statistical model of source traffic. However, they do not take into account traffic dynamic changes. This limitation does not fit well into current designs of networks.

The auction algorithm is an effective model for solving classical assignment problems [6]. Marina Bitsaki et al. pointed out that an auction can outperform substantially its main competitors for important types of problems, both in theory and in practice, and is also naturally well suited for parallel computation.

In addition, Badia et al. studied the connection between resource management and economic parameters, whose application in multimedia communication system is a challenging task [7]. Capone et al. developed an efficient bandwidth allocation algorithm that takes explicitly into account traffic statistics to increase the users' benefit and the network revenue simultaneously [8]. Uday Savagaonkar et al. considered the problem of pricing for bandwidth provisioning over a single link, where users arrive according to a known stochastic traffic model [9]. The network administrator controls the resource allocation by setting a price at every epoch, and each user's response to the price is governed by a demand function.

Pricing has recently attracted a considerable amount of attention as an effective means to achieve economic efficiency in the Internet. A number of pricing schemes have been proposed, such as $[10,11]$. Despite the various strategies presented, the basic idea is that an appropriate pricing policy will provide 
incentives for users to behave in ways that improve overall utilization and performance.

A proper price policy can never be overlooked because it directly influences user demands and network revenue. There are numerous papers on pricing for communication networks using the principles of microeconomics. Kelly et al., for example, use congestion price for rate control in wired networks, where elastic traffic users value only the throughput [12] and can dynamically change their data rates. Siris applies a similar approach to CDMA networks [13]. Low has presented a duality model of several TCP/AQM gate protocols [14]. It interprets these protocols as distributed primaldual algorithms carried out over the Internet in real time to maximize aggregate utility subject to capacity constraints. However, these approaches suffer from their complexity, and are difficult to implement.

In [2], we presented an economic generalized particle model (EGPM) for solving the network bandwidth allocation problem. EGPM is based on OGPM [1] and the excess demand function. It has retained the advantages and overcome the disadvantages of the price-based approaches as mentioned above, as follows.

- It adds congestion-dependent components to the price.

- It takes network activities and dynamic traffic changes into account.

- Its price depends on the network resources' excess demands.

- It does not require resource reservation in the network.

- It uses price to reflect the dynamic situation of resource demands and supply.

- It does not assign the same amount of bandwidth to all classes of traffic.

- Its complexity is relatively lower.

Similar to the auction algorithm, the basic idea of EGPM is that an appropriate pricing policy will provide incentives for users to behave in ways that improve overall utilization and performance.

\section{LIMITATIONS OF OGPM}

EGPM [2] is a variation of OGPM [1] which uses two force-fields: demand force-field and resource force-field. There are numerous particles and forces in every force-field, with each particle following its own dynamic equations to represent network entities, and each force following its own time-varying properties to represent various social interactions among network entities. A particle in OGPM can move along a specific trajectory under the exertion of a composite force.

The OGPM approach features high parallelism, low computation complexities, and easiness for hardware implementation. However, the OGPM approach when applied to the network bandwidth allocation problem has these limitations:

1) The demand force-field in OGPM is used to produce pricing policy. Since prices of bandwidth computed in parallel based on the demand force-field are linked to many factors such as congestion, interaction, resources, demands, etc., the convergence rate of the pricing policy to the equilibrium prices tend to be slow.

2) Since many parameters in OGPM need to be correctly chosen, OGPM can suffer from inefficiency in resource utilization. In order to accommodate as many connections as possible in a congested network, the resources need to be used efficiently. OGPM's complex solutions (in both pricing and resource allocation) appear to be impractical although theoretically they can achieve better resource utilization.

3) With OGPM, once the network traffic changes dynamically, pricing policy is modulated by means of several iterative steps and only when these steps are over can the renewed price take effect. Thus, prices modulate slowly along with the changes of network traffic.

These limitations could seriously prevent the application of the OGPM approach to some network bandwidth allocation problems. It is of interest then to search for a solution that would have the nice theoretical results and yet can result in efficient utilization of resources in practice. EGPM describes one such solution.

\section{EGPM}

EGPM is an extended model based on OGPM, which emphasizes the use of pricing as the network control mechanism. For the pricing, it applies the tatonnement process in economics to OGPM. EGPM arises from OGPM but can overcome some of OGPM's deficiencies for the network bandwidth allocation problem.

In [2], we have studied particularly the relationship between price and demands in networks, and presented the detailed EGPM approach which exploits the price-demands trade-off in solving the bandwidth allocation problem.

There are some common points between EGPM and OGPM.

1) By the two approaches, resource allocation problems in networks are transformed into the kinematics and dynamics of numerous generalized particles in a forcefield.

2) The two approaches regard network links as a kind of generalized particles located in a resource force-field.

3) All the particles evolve simultaneously. And when they arrive at their equilibrium positions, we obtain a feasible solution.

4) Each particle is being exerted upon simultaneously by the force-field and by the forces that represent interactions with other same kinds of particles.

5) OGPM and EGPM are evolutionary swarm optimization approaches. They have evolutionary allocation policies and pricing policies. Their evolutionary allocation policies are produced by a resource force-field.

6) They have the advantages in terms of the higher parallelism, the ease for hardware implementation, etc.

7) This alternative to traditional approaches can deal with a variety of complicated social interactions and autonomous behaviors occurring in networks. 
We summarize the major differences between EGPM and OGPM as follows.

1) OGPM: The pricing policy and the allocation policy are produced by two reciprocal duality force-fields (resource force-field and demand force-field), respectively. EGPM: The resource force-field is retained for allocating network resources; but instead of the demand force-field, the economic tatonnement process is used to produce the pricing policy.

2) OGPM: By defining a Lyapunov function, the convergence of all the particles to their stable equilibrium points is proved. EGPM: We first prove that the iterative price modulation process proposed in [2] converges to equilibrium with certain conditions. Then, we prove that the solution obtained by the EGPM algorithm is globally Pareto optimal.

3) Since the EGPM algorithm is simpler than OGPM in terms of the initialization of the initial conditions and the choice of the main parameters in the algorithm, it is more practical.

4) EGPM is more effective in resource utilization than OGPM, because it introduces the economic tatonnement process in OGPM; what is more, the convergence rate using the EGPM is faster.

5) By EGPM, since only the value of the excess demand function is used in updating the prices, the process has minimal informational requirements, and as such, price modulation works faster than OGPM in response to dynamic network traffic changes.

OGPM and EGPM are evolutionary swarm optimization approaches. However, they have different evolutionary pricing policies, which is a major difference between the two approaches.

The evolutionary pricing policy of OGPM is derived from the partial differential equation taken by the hybrid energy function for the price variable. With OGPM, the price increment of the iterative price modulation process is

$$
\Delta p_{i k}^{(j)}(t) \approx \mathrm{d} p_{i k}^{(j)}(t) / d t=-\frac{\mathrm{d} \Gamma_{i k}^{(j)}(t)}{\mathrm{d} p_{i k}^{(j)}(t)}
$$

The evolutionary pricing policy of EGPM is derived from the excess demand function. EGPM's price increment is

$$
\Delta p_{k}^{(j)}(t)=z_{j}(t) \cdot \theta
$$

The iterative price modulation process is

$$
p(t+1)=p(t)+\Delta p(t)
$$

\section{COMPARISON OF OGPM AND EGPM}

In Fig. 1, we show an example of the difference between demand and allocation in a dynamic environment. In Fig. 1(a), the curved line corresponds to the demand and the rectangles the supply (allocated bandwidth) over a period of time. Let $T$ denote the time to calculate the equilibrium allocation in each iteration. Because the allocation does not change until the calculation achieves the equilibrium in the OGPM algorithm, the allocation stays unchanged between $A(t)$ and $B(t)$ in the figure. It needs time $T$ to reach equilibrium and the allocation is based on the old environment, at $A(t-T)$.

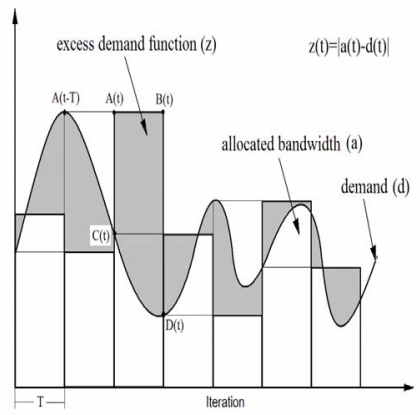

(a) OGPM

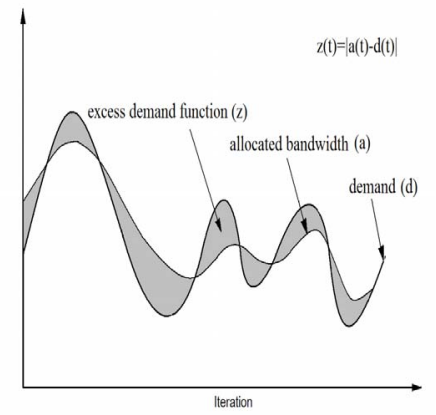

(b) EGPM
Fig. 1 The excess demand generated by the difference between the allocation and the environment

EGPM allows links to trade resources to settle excess demand at every iteration. In Fig. 1(b), the two curved lines represent the change of the demand and the allocated bandwidth, respectively. For both cases, the shadowed regions represent the "excess demand function" (i.e., over- or underallocation). The shaded regions of both OGPM and EGPM shrink when the interval of environmental changes becomes longer. When that interval is small the excess demand function of EGPM is small, in which case EGPM is preferable. On the other hand, when the interval of environmental change is large the excess demand function of OGPM is small and OGPM is preferable, because the time to reach the equilibrium by oneby-one exchange (i.e., renewed allocation at every iteration step) is larger than that by OGPM.

Next, we compare the satisfaction ratio which is defined as $\left(\sum_{k=1}^{n_{j}} a_{k}^{(j)}(t)\right) / d^{(j)}(t)$. There is a direct relationship between the satisfaction ratio and the loss of the the excess demand function is large and the allocation is not preferable, and if the ratio is large, the loss is small and the allocation is well done. The ratio becomes 1 if and when the allocation matches exactly the demand.

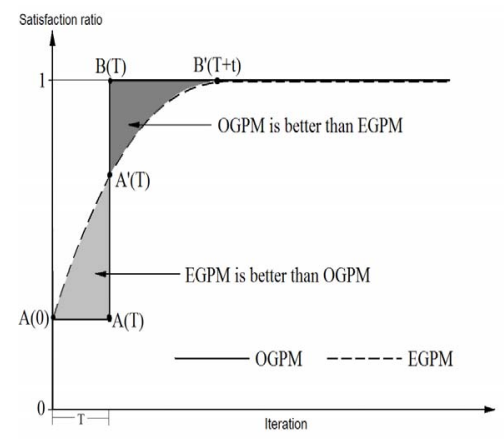

Fig. 2 The satisfaction ratio of demand in static environment

In a static environment where the demands of the paths do 
not change, the satisfaction ratio changes in a fashion which is as illustrated in Fig. 2. At regions $A(0), A(T)$, and $A^{\prime}(T)$ in the figure, OGPM cannot improve the quality of allocation because the calculation has not finished. On the other hand, EGPM gives renewed allocations in these regions, which is desirable. In regions $A^{\prime}(T), B(T)$, and $B^{\prime}(T+t)$, OGPM reaches equilibrium and the allocation by OGPM is better than that by EGPM. In reality, for a static environment, when $T$ is small, OGPM is preferable, and when $T$ is large, EGPM is preferable.

In a dynamic environment, the changes of the satisfaction ratio are illustrated in Fig. 3 by an example. In Fig. 3(a), because the environment changes soon after the OGPM reaches equilibrium or before the OGPM reaches equilibrium, the allocation by OGPM is effective only for short bursts of time. On the other hand, in Fig. 3(b), the allocation by EGPM adapts better to the changing environment. That is, most of the time, EGPM is more effective in allocating bandwidth than OGPM in dynamic evironments.

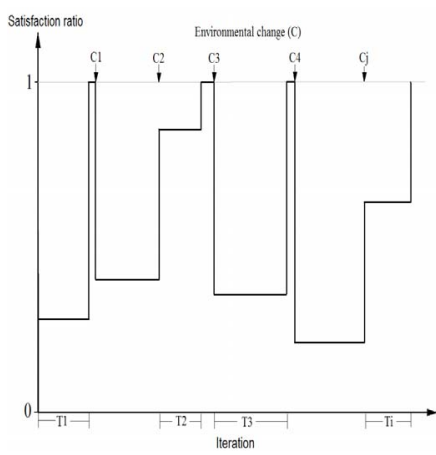

(a) OGPM

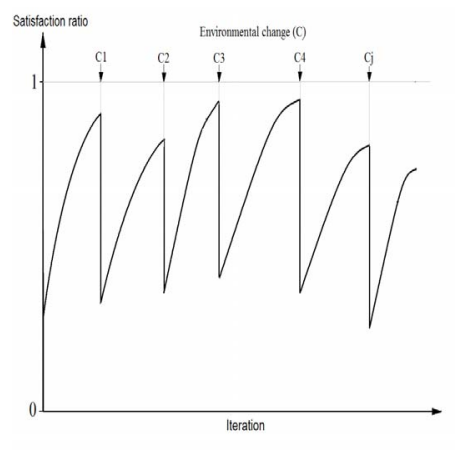

(b) EGPM
Fig. 3 The satisfaction ratio of demand in dynamic environment

\section{COMPARISON THE TWO MODELS WITH OTHER APPROACHES}

In order to compare the effectiveness of the algorithms OGPM and EGPM on a broader scale, we add to the comparison the Lagrangrian multiplier approach, the max-min algorithm, and ant colony algorithm. Their relative differences are summarized in Table 1.

For comparing their effectiveness in allocation and how well they satisfy the demands, we conduct two experiments for a static and a dynamic environment respectively. For both environments, there are 20 links and 20 paths in the network. We run OGPM, EGPM, Lagrangrian multiplier, Ant colony and Max-min algorithms five times with different parameter settings. The source code of Ant colony optimization is from URL: http://www.geocities.com/saurabhsamdani /sourcecodes.html.

The max-min theorem is particularly useful in deriving a practical method for obtaining a max-min fair allocation, called "progressive filling" [25, page 11]. The idea is as follows. We start with all rates being equal to 0 and grow all rates together at the same pace, until one or several link capacity limits are hit. The rates for the sources that use these links are not increased any more, and we continue increasing the rates for other sources. All the sources that are stopped have a bottleneck link. This is because they use a saturated link, and all other sources using the saturated link are stopped at the same time, or were stopped before, thus having a smaller or equal rate. The algorithm continues until it is not possible to increase.

The Lagrangrian multiplier algorithm requires solving some constrained optimization problem. We use some functions such as linear programming function "lp()" of MATLAB to get the experiment results. In the experiments, the utility function and constraint conditions are defined according to [15, page 12 14].

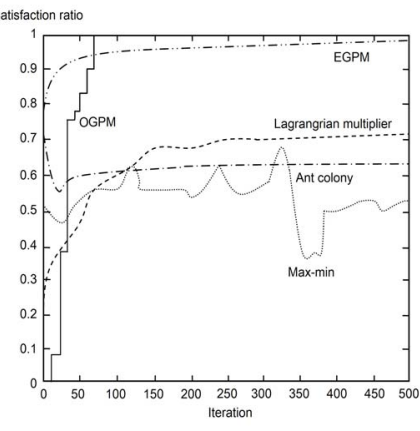

(a) Static environment

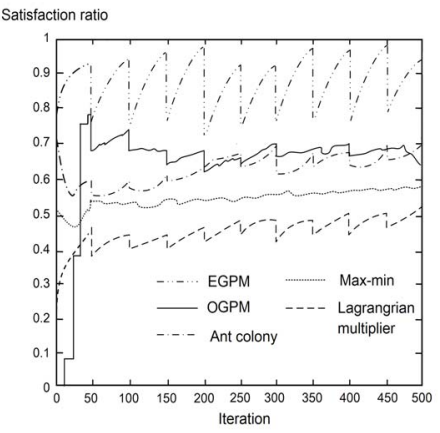

(b) Dynamic environment
Fig. 4 The satisfaction ratio of different approaches

The results of the first experiment for the static case are shown in Fig. 4 (a). With OGPM, the satisfaction ratio of demand rises abruptly and intermittently. It is because the shown satisfaction ratio is the average of the results in many experiments. In fact, for a single instance of allocation, the satisfaction ratio would become 1 at the end of the OGPM calculation.

In Fig. 4 (a), the satisfaction ratio of demand improves gradually for EGPM, even at early stages of the calculation; this is because of the one-by-one exchange feature of EGPM. When there are many links and resource is much more than the demand of paths, the satisfaction ratio of demand is about 95\% (as shown in Fig. 4 (a)). When there are only a few links and resource is less than the demand of paths, the satisfaction ratio of demand also improved remarkably at early stage of calculation, but the elapsed time is relatively long.

In the early stage (iterations) of the experiment using OGPM, the calculation did not converge and some initial allocated bandwidth (i.e., (a)) was used as allocation. The satisfaction ratio of demand did not improve considerably because the allocation was not changed. Only a little increase of the ratio happened because the allocation is computed by the price at that time. On the other hand, when equilibrium allocation was obtained, the allocation agreed with the demand and the satisfaction ratio of demand became 1 . The time to calculate the allocation was about 70 in the case that the resource of links is much more than the demand of paths.

As can be seen in Fig. 4 (a), the satisfaction ratio of demands 
Table 1 EGPM vs. OGPM and other well-known algorithms

\begin{tabular}{|c|c|c|c|c|c|}
\hline & EGPM & OGPM & Lagrangrian multiplier & Ant colony & Max-min \\
\hline $\begin{array}{l}\text { Adaptive to } \\
\text { topology changes }\end{array}$ & Fast & Middle & Slow & Middle & Fast \\
\hline Routing overhead & Low & Low & High & Low & Low \\
\hline $\begin{array}{l}\text { Building routing } \\
\text { preference }\end{array}$ & $\begin{array}{c}\text { By Excess } \\
\text { demand function }\end{array}$ & $\begin{array}{c}\text { By Hybrid } \\
\text { energy functions }\end{array}$ & $\begin{array}{l}\text { By Utility } \\
\text { function }\end{array}$ & $\begin{array}{c}\text { By Pheromone } \\
\text { laid }\end{array}$ & $\begin{array}{c}\text { By Transmission } \\
\text { time/delay }\end{array}$ \\
\hline
\end{tabular}

of the Lagrangrian multiplier approach is from about 0.3 to about 0.7 , and the increase is monotonous but steady. The results are consistent with [16]. We also obtained results for the max-min approach for the satisfaction ratio, which are shown as the dotted line in Fig. 4. The ratio fluctuates between 0.4 and 0.6. The satisfaction ratio of the demand of the ant colony approach is around 0.6.

Next, we show the results of the second experiment. We set the interval of change to 50 time units. We arrange the resources of the links to be almost equal to the path demands. As an environmental change, we adopt the change of the satisfaction ratio of demand. We show only the satisfaction ratio of one path and the results shown below in Fig. 4 (b) are those of that chosen path.

When changes happen every 50 time units, the allocation by OGPM cannot completely measure up the paths' preference because OGPM needs about 70 time units to reach equilibrium. On the other hand, with one-to-one exchange based on EGPM, although the satisfaction ratio drops at the change of environment, the ratio increases monotonously until the next environmental change.

For a fast changing environment, the Lagrangrian multiplier approach appears to have problem keeping up, as can be seen in Fig. 4 (b). The ant colony and the max-min approach are more adaptive to environmental changes, and their satisfaction ratio did not deteriorate as remarkably in the same dynamic environment.

We conclude that, from the results of the two experiments (Fig. 4 (a) and Fig. 4 (b)), EGPM is superior to the other three bandwidth allocation approaches in static and dynamic environments; and EGPM is superior to the OGPM approach in dynamic environments.

\section{CONCLUSION}

In this paper, we compare EGPM with OGPM in static and dynamic environments, and compare them also with the ant colony optimization approach, the utility function algorithm and the max-min fairness algorithm.

EGPM is an important extension and further development of OGPM. In a static environment, if the time to calculate the equilibrium allocation in each iteration is small, OGPM is preferable, and if the time is large, EGPM is preferable.

In addition, EGPM has better adaptive ability in a real-time dynamic network environment than OGPM.

\section{Acknowledgement}

This project is supported in part by the Hong Kong General Research Fund (HKU 713708E).

\section{REFERENCES}

[1] Dianxun Shuai, Xiang Feng. The Parallel Optimization of Network Bandwidth Allocation Based on Generalized Particle Model. Computer Networks. 2006, 50(9): 1219-1246.

[2] Xiang Feng, Francis C. M. Lau, Dianxun Shuai. A Nature-Inspired Algorithm for Intelligent Optimization of Network Resources. IEEE International Conference on Communications Systems (ICCS 2008). Accepted. Paper $\sharp 1569139809$.

[3] X. Wang and H. Schulzrinne, Pricing Network Resources for Adaptive Applications in a Differentiated Services Network Infocom, 2001

[4] Baglietto M.1; Bolla R.; Davoli F.; Marchese M.; Mongelli M. A proposal of new price-based Call Admission Control rules for Guaranteed Performance services multiplexed with Best Effort traffic. Computer Communications, Volume 26, Number 13, 15 August 2003, pp. 1470$1483(14)$

[5] Jin, N; Jordan, S. The effect of bandwidth and buffer pricing on resource allocation and QoS. Computer Networks. Vol. 46, no. 1, pp. 53-71. Sept. 2004

[6] Bitsaki, M. Stamoulis, G.D. Courcoubetis, C. Auction-based bandwidth trading in a competitive hierarchical market. IEEE International Conference on Next Generation Internet Networks. 18-20 April 2005: 372379

[7] Badia, L; Lindstroem, M; Zander, J; Zorzi, M. An economic model for the radio resource management in multimedia wireless systems. Computer Communications. Vol. 27, no. 11, pp. 1056-1064. July 2004

[8] A. Capone, J. Elias, F. Martignon, G. Pujolle. Dynamic Resource Allocation in Communication Networks. IEEE Trans. on Networking. 2006

[9] Savagaonkar, U; Chong, E K P; Givan, R L. Online pricing for bandwidth provisioning in multi-class networks. Computer Networks. Vol. 44, no. 6, pp. 835-853. Apr. 2004

[10] Rich Wolski, James S. Plank, John Brevik, Todd Bryan. Analyzing Market-Based Resource Allocation Strategies for the Computational Grid. International Journal of High Performance Computing Applications. Volume 15, Issue 3, August 2001 : 258 - 281

[11] Panagiotis Thomas, Demosthenis Teneketzis, Jeffrey K. MackieMason. A Market-Based Approach to Optimal Resource Allocation in Integrated-Services Connection-Oriented Networks. Operations Research. Volume 50, Issue 4, 2002: 603-616

[12] F. P. Kelly, A. K. Maullo, and D. K. H. Tan, "Rate control in communication networks: Shadow prices, proportional fairness and stability," J. Oper. Res. Soc., Vol. 49, pp. 237-252, 1998.

[13] Vasilios A. Siris, "Resource control for elastic traffic in CDMA networks," Proceedings of the 8th annual international conference on Mobile computing and networking, Atlanta, USA, pp. 193 - 204, 2002.

[14] S. H. Low. A Duality Model of TCP and Queue Management Algorithms. IEEE/ACM Transactions on Networking, August 2003, 11(4):525-536

[15] Lijun Chen, Steven H. Low, Mung Chiang, John C. Doyle. Cross-layer Congestion Control, Routing and Scheduling Design in Ad Hoc Wireless Networks. IEEE INFOCOM, 2006. $<$ http://netlab.caltech.edu/pub/papers/wireless-Chen-infocom06.pdf >

[16] Jean-yves LE Boudec. Rate adaptation, Congestion Control and Fairness: A Tutorial. November 22, 2005. <http://icawww1.epfl.ch/cn2/0506/slides/LEB- 3132.pdf> 